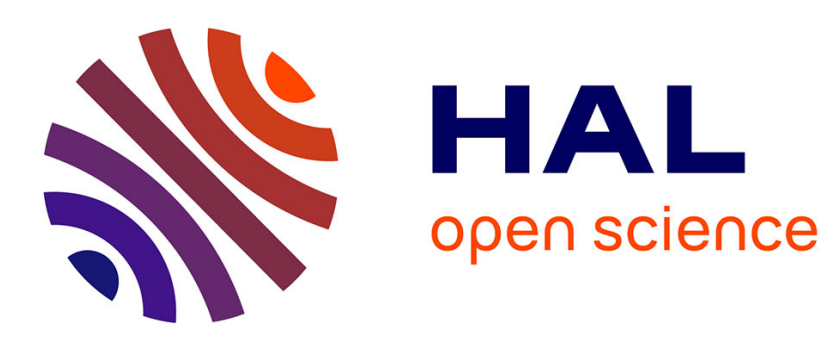

\title{
Croissance épitaxique de composés semiconducteurs par évaporation-diffusion en régime isotherme
}

\author{
G. Cohen-Solal, Y. Marfaing, F. Bailly
}

\section{To cite this version:}

G. Cohen-Solal, Y. Marfaing, F. Bailly. Croissance épitaxique de composés semiconducteurs par évaporation-diffusion en régime isotherme. Revue de Physique Appliquée, 1966, 1 (1), pp.11-17. 10.1051/rphysap:019660010101100 . jpa-00242675

\section{HAL Id: jpa-00242675 https://hal.science/jpa-00242675}

Submitted on 1 Jan 1966

HAL is a multi-disciplinary open access archive for the deposit and dissemination of scientific research documents, whether they are published or not. The documents may come from teaching and research institutions in France or abroad, or from public or private research centers.
L'archive ouverte pluridisciplinaire HAL, est destinée au dépôt et à la diffusion de documents scientifiques de niveau recherche, publiés ou non, émanant des établissements d'enseignement et de recherche français ou étrangers, des laboratoires publics ou privés. 


\title{
CROISSANCE ÉPITAXIQUE DE COMPOSÉS SEMICONDUCTEURS PAR ÉVAPORATION-DIFFUSION EN RÉGIME ISOTHERME (1)
}

\author{
Par G. COHEN-SOLAL, Y. MARFAING et F. BAILLY, \\ Laboratoire de Magnétisme et de Physique du Solide, C. N. R. S., Bellevue, Seine-et-Oise.
}

\begin{abstract}
Résumé. - On présente un nouveau procédé permettant de réaliser l'épitaxie de composés semiconducteurs tels $\mathrm{HgTe}, \mathrm{GeTe}, \mathrm{GaSb}, \ldots$ en régime isotherme, presque à l'équilibre thermodynamique. Des couches monocristallines d'épaisseur 1 à $200 \mu$ ont été obtenues. Le phénomène de transport consiste en une évaporation de matière de la source au substrat, couplée à une diffusion dans le substrat des atomes transférés. Les paramètres de préparation étudiés sont la distance de la source au substrat, la durée et la température du traitement. La variation de l'épaisseur du dépôt avec la température fait apparaître, aux hautes températures, une énergie d'activation de $29 \mathrm{kc} / \mathrm{mole}$ en bon accord avec les prévisions théoriques.
\end{abstract}

Abstract. - A new method is described for epitaxial growth of semiconducting compounds such as HgTe, GeTe, GaSb, ..., in an isothermal system, approaching thermodynamic equilibrium. Single crystal layers 1 to $200 \mu$ thick have been obtained. The transport is assumed to proceed via evaporation from the source onto the substrate, associated with a diffusion of the impinging atoms within the substrate. The growth parameters investigated are : spacing between source and substrate, time and temperature of treatment. An activation energy of $29 \mathrm{kc} / \mathrm{mole}$, consistent with the theoretical assumptions, has been determined from the variation of the layer thickness with temperature.

1. Introduction. - Le développement de nouveaux dispositifs électroniques provoque depuis quelques années de nombreuses études sur la croissance de films monocristallins de matériaux semiconducteurs; différentes méthodes de croissance épitaxique ont été proposées à cet effet. Les techniques usuelles sont souvent d'application délicate et limitéa et utilisent des appareillages complexes. Presque toujours, les conditions de mise en œuvre impliquent un contrôle rigoureux de paramètres tels que : des débits gazeux, des flux partiels de constituants, des gradients de température.

La méthode nouvelle que nous présentons ici se distingue de celles qui ont été décrites à ce jour, en ce que la croissance épitaxique est obtenue par un traitement isotherme. Nous l'appellerons Evaporation-Diffusion en Régime Isotherme (E. D. R. I.).

2. Principe. - La possibilité d'un transport net de matière en régime isotherme avait été suggérée par l'observation d'un déplacement anormal de rainures au cours de l'interdiffusion à l'état solide des cristaux de HgTe et CdTe [1],

La méthode proposée dérive de ces expériences : elle consiste à placer face à face, à courte distance, deux échantillons de matériaux différents, et à porter l'ensemble à une température donnée. Le flux de matière émis par le corps le plus volatil (source)

(1) Cette étude a été effectuée dans le cadre d'un contrat de recherches avec la D. R. M. E. rencontre la surface de l'autre corps (substrat); à cette surface prend naissance un flux de diffusion, vers l'intérieur du substrat, sous l'action d'un gradient de concentration. Une couche de la matière constituant la source est progressivement construite sur le substrat, tandis que se poursuit une diffusion mutuelle entre la matière du substrat et celle de la couche. Ces mécanismes seront précisés plus loin, mais il apparaît dès l'abord qu'ils sont conditionnés par la valeur de paramètres thermodynamiques caractérisant le couple de matériaux (énergies de sublimation, coefficients de diffusion, etc...).

Comme la méthode dite de "close-spacing " ou " sandwich ", qui a permis entre autres la réalisation de dépôts épitaxiques de GaAs sur Ge [2] ou de silicium dopé sur silicium [3], la méthode E. D. R. I. a l'avantage d'un rendement en matière élevé : en maintenant à proximité immédiate la source et le substrat, on favorise la recombinaison stoechiométrique du composé volatil sur la surface exposée, et le rapport de la masse déposée à la masse évaporée est très voisin de l'unité. Mais son caractère isotherme distingue la méthode $\mathrm{E}$. D. R. I. de celle du "sandwich" et lui confère une grande simplicité et d'autres avantages qui seront discutés plus loin.

3. Mode opératoire. - On opère en tube de quartz, rincé à l'argon, vidé jusqu'à une pression dynamique d'environ $10^{-3} \mathrm{~mm}$ de mercure, puis scellé. Le tube de quartz est choisi de telle sorte 
qu'il puisse résister aux pressions relativement élevées qui se développent en cours d'opération.

A l'intérieur de ce tube est disposée une nacelle en graphite pur, dégazée sous vide, renfermant le cristal-source et le cristal substrat; ces derniers sont maintenus à une distance connue - pouvant varier de 100 à 2000 microns - par des cales de silice ou de molybdène. Un couvercle en graphite, pressé par de petits ressorts de molybdène, ferme la nacelle.

Le tube de quartz ainsi équipé est introduit au sein d'une masselotte de cuivre de masse thermique élevée, qui a un double rôle : d'une part elle évite une variation importante de la température du four quand on introduit le tube, d'autre part elle homogénéise la température de façon que l'ensemble ainsi constitué, placé dans la région de température uniforme d'un four à thermostat, soit bien isothëríme.

Dans ces conditions, le dépôt s'effectue principalement sur la face en regard de la source, et prend la forme délimitée par celle des cales utilisées. On observe toutefois sur la face externe du substrat un léger dépôt, d'épaisseur généralement faible. Sur les tranches, il se développe parfois, en des centres particulièrement propices à la nucléation, de gros cristaux de forme tétraédrique ou pyramidale.

En fin d'opération, le tube est extrait du four et subit une trempe à l'eau.

4. Application de la méthode E. D. R. I. au couple HgTe-CdTe. - On a récemment démontré la possibilité d'utiliser le couple de composés semiconducteurs II-VI, HgTe-CdTe, dans la préparation de structures à largeur de bande interdite variable, capables de détecter le rayonnement infrarouge. Dans la phase initiale de recherche, ces structures étaient préparées par interdiffusion à l'état solide [4], [5], [6]. La méthode E. D. R. I. nous fournit ui deuxième procédé de préparation plus souple. C'est pour cette raison pratique que le couple $\mathrm{HgTe}-\mathrm{CdTe}$ a été utilisé pour effectuer les expériences détaillées sur cette méthode, dont nous rendrons compte maintenant. Ce couple est d'ailleurs, a priori, particulièrement favorable : d'abord parce que les deux composés cristallisent dans la même structure (blende), avec des paramètres de mailles différant de moins de $0,3 \%$, et forment des solutions solides continues $\mathrm{Cd}_{\mathbf{x}} \mathrm{Hg}_{1-x} \mathrm{Te}$ [7] ; en second lieu parce que leurs tensions de vapeur sont différentes, de sorte qu'on peut admettre que la pression totale à l'intérieur du système isolé est presque égale à la pression partielle du tellurure de mercure. Cette dernière pourrait être, si nécessaire, contrôlée indépendamment de la température de l'ensemble de la source et du substrat par la pression de vapeur de mercure au point froid, dans un système à deux températures.

On a déjà rendu compte [8] de résultats préliminaires sur l'application de la méthode E. D. R. I. au cas de substrats monocristallins de CdTe, orientés normalement à l'axe polaire, résultats montrant que l'épitaxie était obtenue.

Préparation des échantillons. - La source, constituée par HgTe pur, est choisie indifféremment sous forme poly ou mono-cristalline. Le substrat de tellurure de cadmium est par contre un monocristal. Purifiés par fusion de zone et cristallisés par la
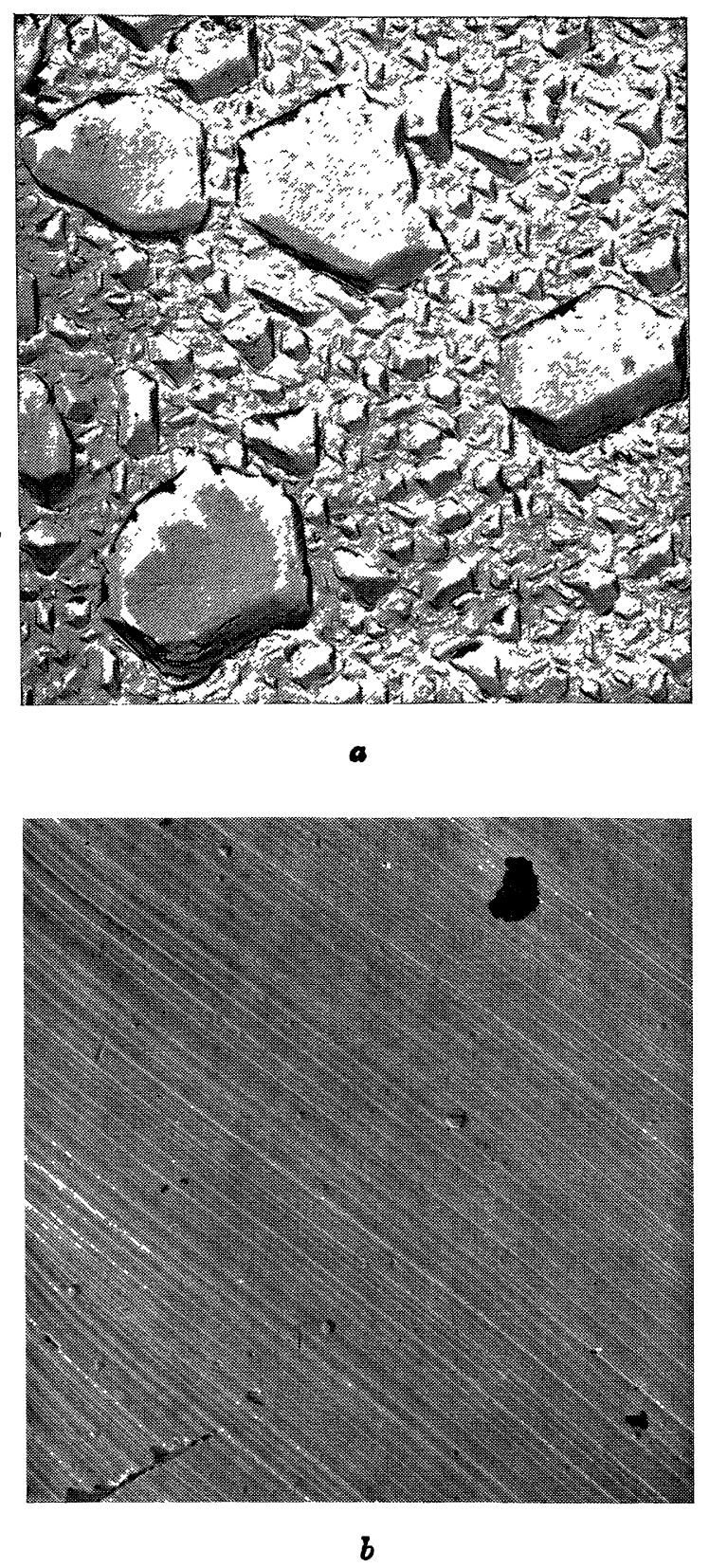

FIg. 1. - Surface d'une couche monocristalline de Hg'Te déposée sur un plan (111) d'atomes de cadmium, d'un cristal de CdTe : $1 a$ ) dépôt effectué à $T<450^{\circ} \mathrm{C}$ et $1 b)$ à $T>450{ }^{\circ} \mathrm{C}$. 
méthode de Bridgman, ces matériaux nous ont été fournis par H. Rodot et R. Triboulet.

Les cristaux de dimensions $8 \times 4 \times 2 \mathrm{~mm}^{3}$, subissent un polissage mécanique ; un "poli optique" est donné à l'une de leurs grandes faces. Après un nettoyage au trichloréthylène bouillant, suivi d'un rinçage à l'alcool isopropylique, on les soumet à un traitement de polissage chimique au moyen de solutions appropriées [9]. Un rinçage soigné à l'eau désionisée termine ces opérations.

La surface, " propre " des points de vue chimique et cristallographique, est rarement plane et lisse. Le plus souvent on observe de légères ondulations, avec une attaque plus importante sur les bords et le long de lignes ou de points d'émergence de dislocations.

Aspect des couches déposées. - Observées au microscope optique, les couches déposées sur un substrat monocristallin d'orientation quelconque présentent un aspect assez plat, légèrement " grenu ", un peu à la manière d'une peau d'orange.

Les différences d'aspect liées à la polarité du substrat (orientation 111 ou 111) ont été présentées antérieurement [8]. La figure 1 représente quelques aspects de telles couches.

Étude de la structure. - Dans tous les cas, les diagrammes de diffraction de rayons $\mathrm{X}$ (Laue) révèlent une parfaite monocristallisation des couches déposées, quelle que soit l'orientation cristalline du support et quelle qu'en soit la nature superficielle dans les cas d'orientation (111) ( $f g$. 2). La couche

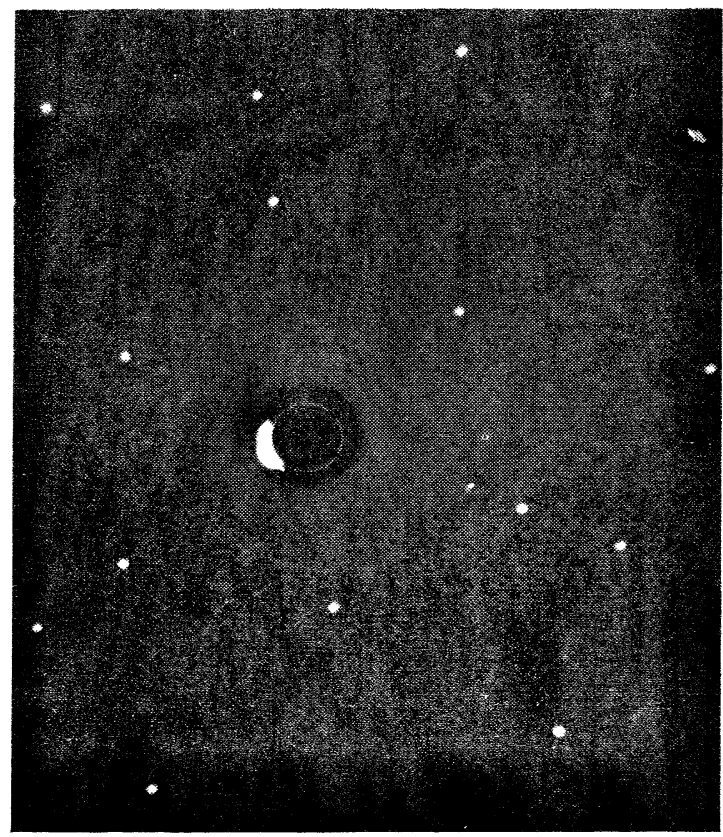

Fig. 2. - Diagramme de Laue d'une couche épitaxique de HgTe sur CdTe (111). conserve, au cours de sa croissance, la structure et l'orientation du substrat. Ceci peut être observé sur la microphotographie de la surface d'un dépôt reproduisant avec fidélité une macle du substrat (fig. 3).

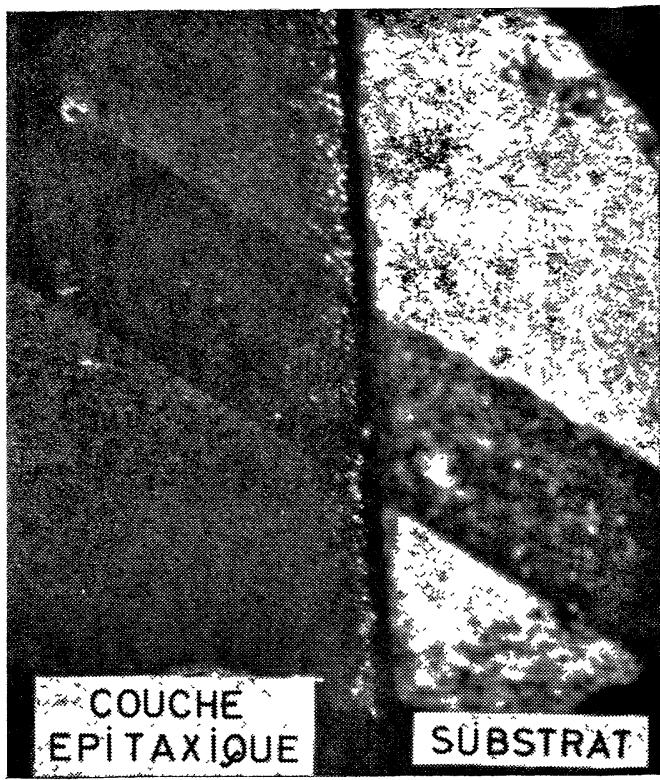

Fig. 3. - Macle d'un substrat de CdTe, reproduite par une couche épitaxique de HgTe.

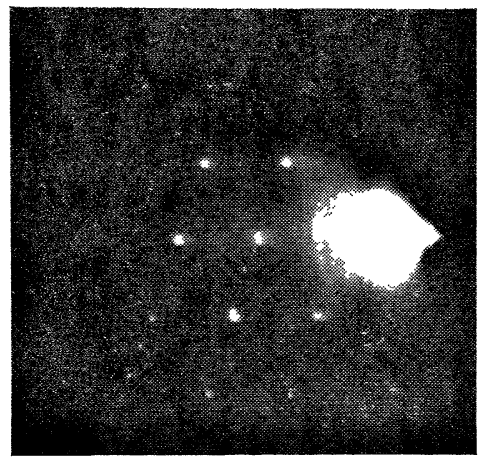

Fig. 4. - Diagramme de diffraction d'électrons en réflexion sur une couche de $\mathrm{HgTe}$ déposée sur un cristal de CdTe.

En diffraction d'électrons, de telles couches ont fait apparaître des lignes de Kikuchi intenses. La figure 4 présente un diagramme typique de diffraction par réflexion et la figure 5 , un diagramme obtenu par transmission. (Les points supplémentaires qui apparaissent en dehors de la structure simple suggèrent l'existence de macles dans ce cas particulier.)

INFLUENCE DES DIVERS PARAMÈTRES DU SYSTÈME SUR L'ÉPAISSEUR DES DÉPOTS. - Les épaisseurs des couches obtenues dépendent d'un grand 


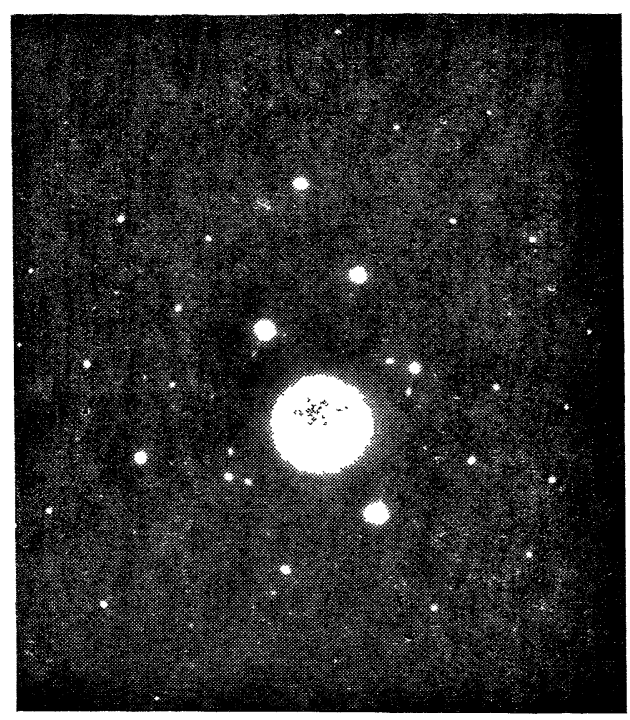

FIG. 5. - Diagramme de diffraction d'électrons par transmission (HgTe sur CdTe).

nombre de paramètres. Certains d'entre eux ont été maintenus à une valeur constante; les plus importants on fait l'objet d'une étude systématique : ce sont en particulier la durée du traitement, la distance entre source et substrat et la température.

Le rôle de deux autres paramètres, l'orientation cristallographique du substrat ainsi que la nature de ses défauts de réseau, est actuellement en cours d'étude. Des premiers résultats, il ressort qu'une certaine différence d'épaisseur apparaît entre les dépôts effectués, à basse température, sur des substrats d'orientation quelconque et ceux effectués sur des substrats orientés normalement à l'axe [111]. Cette différence a tendance à s'atténuer et à disparaître quand on élève la température de traitement.

Quant aux défauts de réseau du substrat, ils interviennent par leur influence sur les mécanismes de diffusion; on sait que leur concentration, et même leur nature, peuvent être affectées par les traitements thermiques subis par le substrat au cours de sa préparation. Pour simplifier notre étude, nous avons pris la précaution de choisir des substrats toujours préparés de façon identique.

INFLUENGE dE LA DURÉ de TRAITEMENT. - On a fait varier la durée de traitement, en maintenant constantes la température du système $\left(550^{\circ} \mathrm{C}\right)$ et la distance séparant la source du substrat (108 microns). De 15 minutes à 120 heures, l'épaisseur du dépôt croît approximativement comme la racine carrée du temps ( $f$ g. 6).

Cette relation, non linéaire, montre que la vitesse de croissance n'est pas constante du début à la fin de l'opération. Aussi avons nous adopté, au cours de notre étude, l'épaisseur du dépôt atteinte au bout

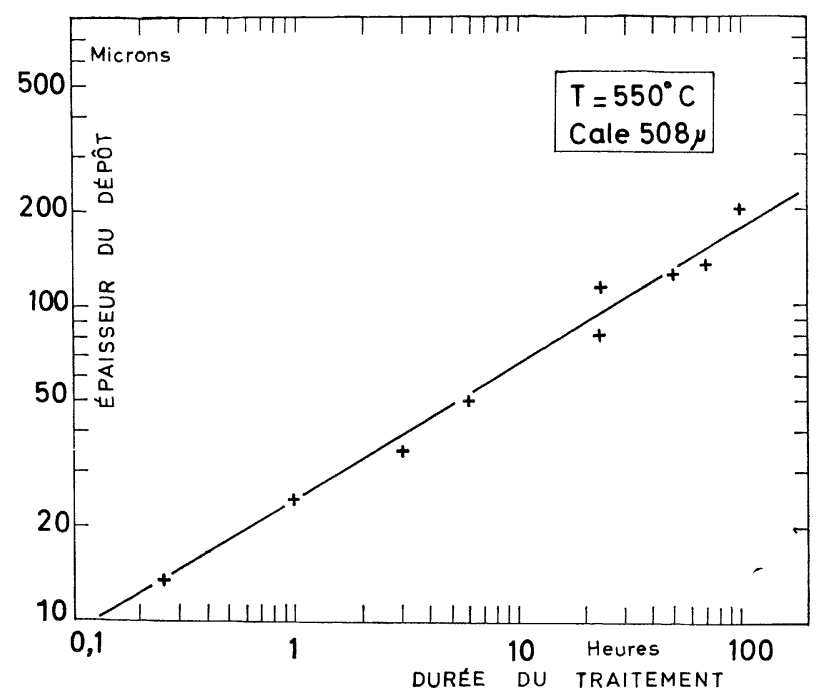

Frg. 6. - Épaisseur du dépôt

en fonction de la durée du traitement.

d'un temps donné, comme grandeur représentant la quantité de matière transportée sur le substrat.

La loi de variation a pour expression :

$$
e=B t^{n}
$$

$(t=$ durée (en heures) du traitement ; $B=25 \mathrm{mi}$ crons (heure) $-\mathbf{0 . 4 2} ; n=0,42)$.

INFLUENGE DE LA DISTANCE SOURCE-SUBSTRAT. L'influence de l'écartement entre la source et le

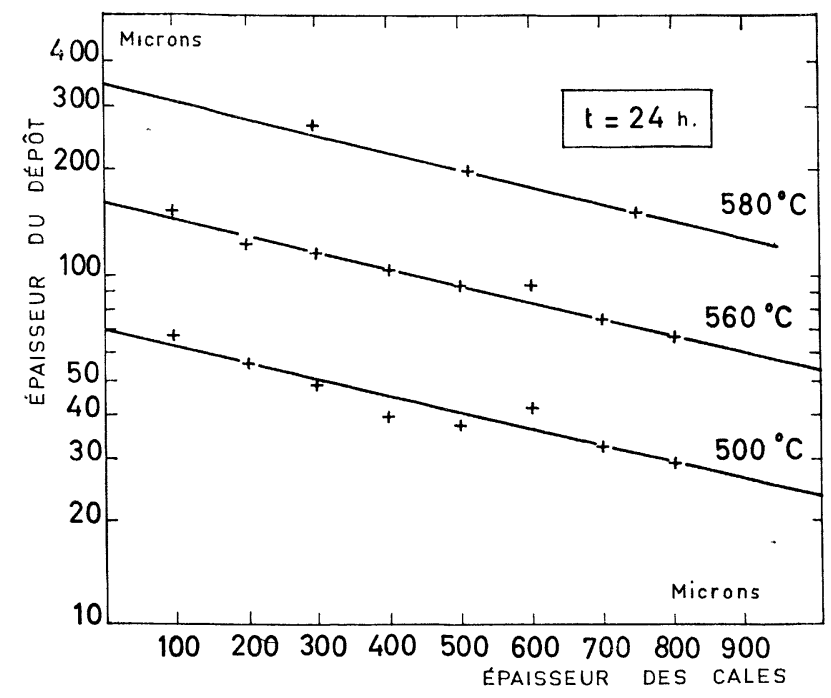

FIG. 7. - Épaisseur du dépôt en fonction de la distance entre la source et le substrat.

substrat est représentée sur la figure 7, pour des températures de $500^{\circ} \mathrm{C}, 560^{\circ} \mathrm{C}$ et $580^{\circ} \mathrm{C}$, et une durée de traitement constante égale à 24 heures. Le 
logarithme de l'épaisseur du dépôt varie de façon linéaire avec cet écartement :

$$
e=C \exp (-\alpha d)
$$

( $e=$ épaisseur du dépôt ; $d=$ distance entre source et substrat (en microns) ; $C=$ respectivement 70 , 160 et 350 microns aux températures de $500{ }^{\circ} \mathrm{C}$ $560^{\circ} \mathrm{C}$ et $580^{\circ} \mathrm{C} ; \alpha=1,12 \times 10^{-3}$ (microns) ${ }^{-1}$ ).

Pour des distances supérieures à celles considérées, il apparaît un redressement des droites qui tendent à devenir parallèles à l'axe des abscisses, et l'épaisseur du dépôt devient pratiquement indépendante de la distance.

INFluence de la température. - La température joue un rôle fondamental dans le mécanisme de croissance. En effet, elle intervient à la fois dans l'établissement des pressions de vapeur, donc du flux d'évaporation, et dans la sélection du processus microscopique régissant la diffusion. Or, comme on l'a montré par ailleurs [1], le couplage du flux d'évaporation et d'un flux de diffusion détermine l'épaisseur du dépôt.

Sur la courbe de la figure 8 , où le logarithme de l'épaisseur du dépôt est portée en ordonnées et l'inverse de la température absolue en abscisses, on a placé des points correspondant à des expériences

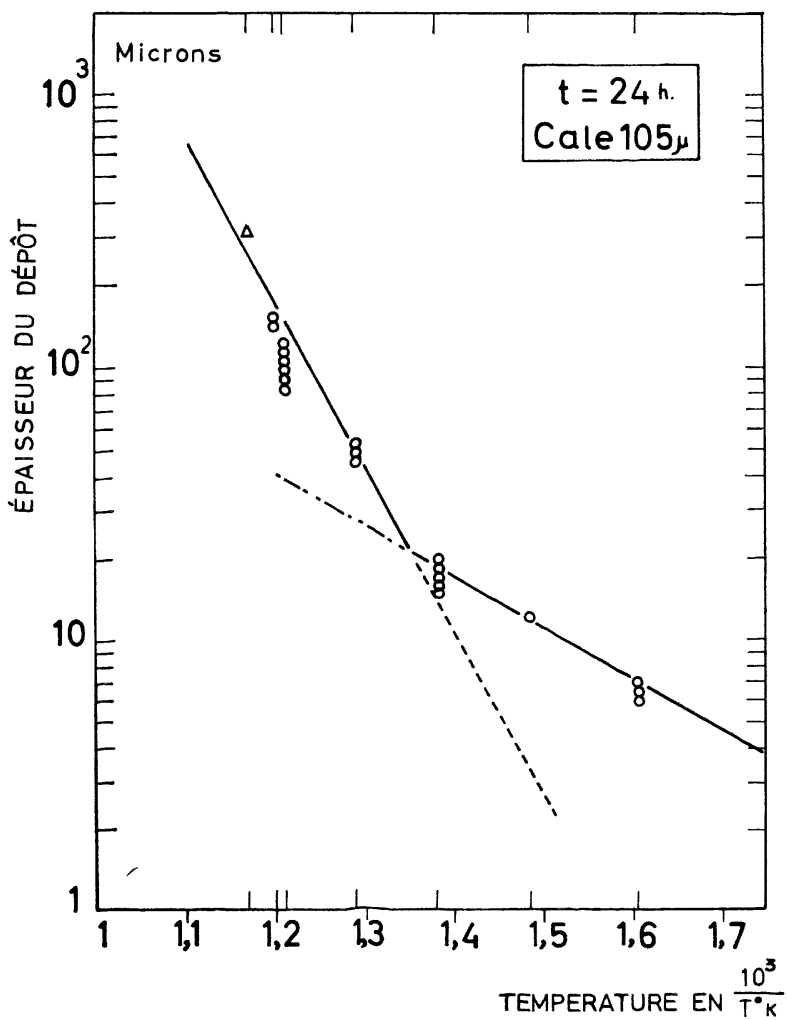

FIG. 8. - Épaisseur du dépôt

en fonction de la température du traitement. effectuées à durée constante $(24 \mathrm{~h})$, pour une dis tance source-substrat maintenue à 108 microns.

Aux pentes des deux droites ont été associées des énergies d'activation. La détermination expérimentale fournit une énergie de $29 \mathrm{Kcal} / \mathrm{mole}$, pour les températures supérieures à $450{ }^{\circ} \mathrm{C}$ environ, et de $9 \mathrm{Kcal} / \mathrm{mole}$ pour les températures inférieures à $450^{\circ} \mathrm{C}$.

L'étude théorique exposée dans l'article [1] avait fait apparaître une énergie d'activation de $28 \mathrm{Kcal} /$ mole pour le domaine de températures situé audessus de $450^{\circ} \mathrm{C}$. L'accord de cette valeur avec la valeur expérimentale est de nature à étayer l'hypothèse émise pour expliquer la migration de rainures au cours d'une interdiffusion [1].

Profil de composition dans les Structures. A l'origine $\mathrm{du}$ processus de croissance se trouve l'interdiffusion. Celle-ci se poursuit pendant toute la durée de l'opération et tout se passe comme si le réseau fictif, constitué par les atomes de tellure du substrat et de la couche déposée, était le siège d'un échange de cations : le mercure migre vers les sites du substrat libérés par le cadmium, qui diffuse vers la couche. Ainsi se constitue au sein de l'ensemble une zone de solution solide de composition générale $\mathrm{Cd}_{\mathbf{x}} \mathrm{Hg}_{1-\mathbf{x}} \mathrm{Te}$; cette composition, constante dans un plan parallèle à la surface initiale, varie d'un plan à un autre.

L'analyse de cette variation de composition a été effectuée au moyen d'une microsonde électronique. Pour cela l'échantillon a été usé sous un angle de $5^{\circ} 4^{\prime}$ dans la zone de croissance, de telle sorte que le pinceau d'électrons puisse explorer successivement la surface initiale (protégée du flux d'évaporation par les cales), la surface du dépôt et le biseau. Ces mesures donnent, avec une précision de l'ordre de $1 \%$ (sur la détermination en poids), la concentration en mercure ; leurs résultats appellent les remarques suivantes:

Le gradient de concentration varie de façon continue et uniforme le long d'une direction normale à la surface : le profil de composition ressemble à celui que l'on obtient par une diffusion à l'état solide [5].

La composition du plan correspondant à la surface initiale est constante, indépendante de la température $\left(T>450^{\circ} \mathrm{C}\right)$ et de la durée du traitement. C'est ce qui caractérise l'interface de Matano, défini dans l'étude de la diffusion intercristalline, et dont l'un de nous [6] a montré qu'il était associé au minimum d'énergie libre du mélange formé par les constituants en présence.

Enfin, il est apparu que l'on obtenait à la surface du dépôt tantôt du tellurure de mercure pur (aux hautes températures), tantôt un alliage de composition intermédiaire (aux basses températures).

Ce résultats est confirmé par des mesures de transmission infrarouge, dont l'exploitation utilise la relation entre la largeur de bande interdite et la compo- 
sition de la couche la plus riche en HgTe de la zone de solutions solides.

Cette observation peut être expliquée en se référant aux différents mécanismes de diffusion susceptibles d'intervenir [1]. Si la diffusion de mercure due au gradient de concentration explique aisément la constitution de la couche d'alliage $\mathrm{Hg}_{1-\mathbf{x}} \mathrm{Cd}_{\mathbf{x}} \mathrm{Te}$ sur le substrat du CdTe, il peut paraître paradoxal de constater que, à haute température, une couche de $\mathrm{HgTe}$ pur se forme en surface et continue à croître quand on prolonge le traitement : on a alors en regard, en effet, deux surfaces de HgTe à la même température, dont l'une crôit aux dépens de l'autre. Mais l'analyse de l'interdiffusion à haute température nous a conduit à admettre la prépondérance d'un mécanisme particulier d'autodiffusion : une diffusion de lacunes de mercure peut expliquer l'apparent paradoxe ci-dessus [1].

La structure d'une couche déposée à haute température $\left(550^{\circ} \mathrm{C}\right)$ est illustrée par la figure 9 , repré-

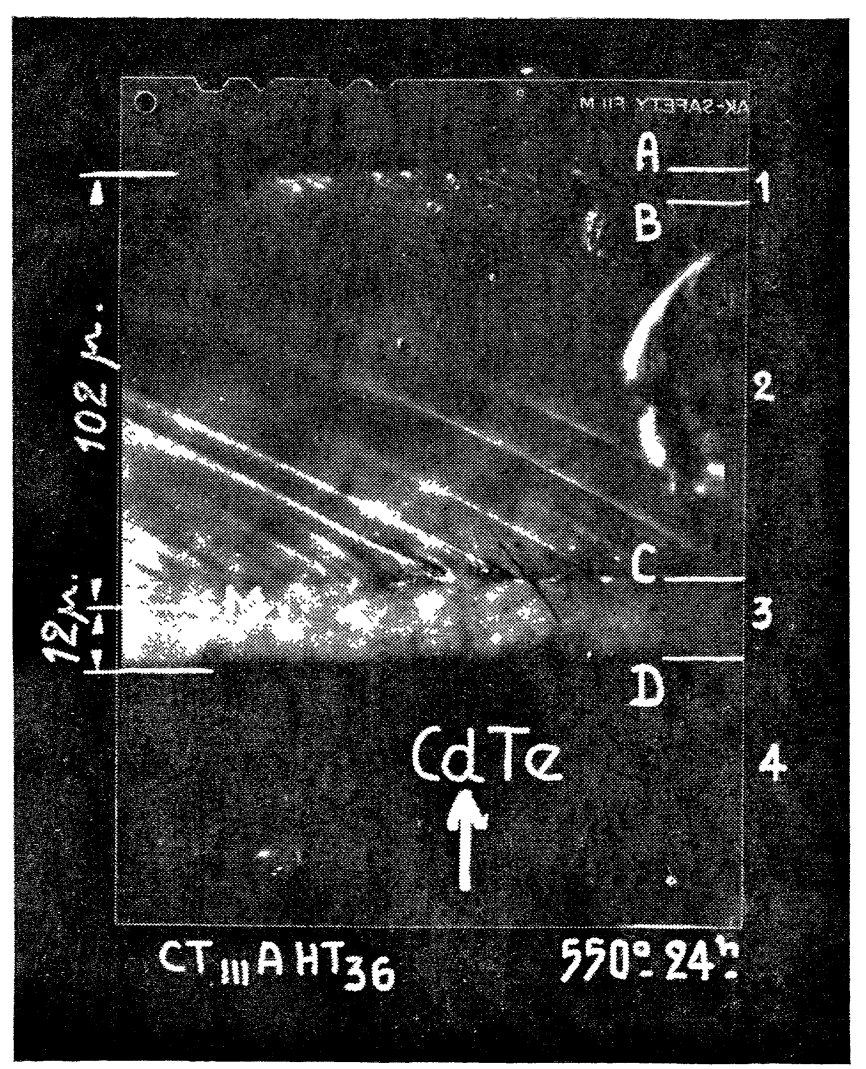

FIg. 9. - Plan de clivage normal

à la surface d'un dépôt de HgTe sur CdTe.

sentant un plan de clivage de l'échantillon, où on distingue trois zones :

- La première, qui s'étend du bordinférieur de la figure jusqu'à une limite marquée $\mathrm{D}$, est constituée par CdTe pur.
- La deuxième, entre les limites $D$ et $B$, est formée par la zone de solutions solides.

- La dernière, qui s'étend de $\mathrm{B}$ au bord supérieur de la couche, notée A, représente $\mathrm{HgTe}$ pur.

On remarque sur la figure la présence de marches de clivage inclinées, prenant naissance dans une ligne de trous $(\mathrm{C})$, qui sépare deux parties de la zone de solutions solides. Il semble que l'on puisse associer cette ligne de trous aux porosités apparaissant en cours de diffusion et habituellement liées à un effet Kirkendall.

Le point $M$ fixe la position de la surface initiale du substrat de CdTe pur, qui est confondue avec celle de l'interface de Matano. Pratiquement, l'épaisseur de la couche déposée est mesurée par $\overline{\mathrm{MA}}$. Dans l'exemple choisi $\left(T=550^{\circ} \mathrm{C} ; t=24 \mathrm{~h}\right.$; cale de $508 \mu$ ), cette épaisseur est d'environ $102 \mu$. Ici encore, l'accord avec l'épaisseur prédite théoriquement $(100 \mu)$ sur la base des coefficients de diffusion connus [5] est excellent.

Conclusion. - L'intérêt de la méthode E. D. R. I réside surtout dans la simplicité de sa mise en œuvre. Les conditions expérimentales sont peu critiques. Pour le couple HgTe-CdTe, l'épitaxie a pu être réalisée dans un intervalle de température $\left(350{ }^{\circ} \mathrm{C}-580{ }^{\circ} \mathrm{C}\right)$ de largeur peu courante. Les épaisseurs de dépôt obtenues ne paraissent pas comporter de limitation et peuvent atteindre facilement quelques centaines de microns.

Une autre caractéristique du procédé est son peu de sensibilité à l'orientation ou à l'état de surface du substrat. C'est ainsi que la qualité cristalline de dépôts effectués sur des supports bruts de polissage mécanique s'est révélée parfaite.

5. Étude d'autres couples de matériaux. - Nous décrirons sommairement quelques expériences préliminaires relatives à d'autres matériaux.

Tout d'abord, outre CdTe-HgTc, nous avons pu réaliser l'épitaxie pour plusieurs couples de matériaux ayant même structure et des paramètres très voisins : dans la structure de la blende, GaSb sur InAs; dans la structure du sel gemme, GeTe sur PbTe. Nous avons aussi obtenu l'épitaxie d'un composé sur un substrat de structure différente ; ainsi HgTe (II-VI, blende) sur PbTe et GeTe (IV - VI, NaCl); GeTe (IV-VI, NaCl) sur CdTe (II - VI, blende).

Une autre application de la méthode a consisté à déposer une solution solide $\mathrm{Cd}_{\mathbf{x}} \mathrm{Hg}_{1-\mathbf{x}} \mathrm{Te}$ sur un substrat de CdTe ou de solution solide de composition différente.

On peut voir, sur la figure 10, l'aspect d'une couche de GaSb déposée sur un substrat de InAs orienté normalement à l'axe [111]. Les zones triangulaires noires sont dues à des parties de la couche arrachées et entraînées sur la réplique. La partie claire donne une vue de la surface elle-même. En 


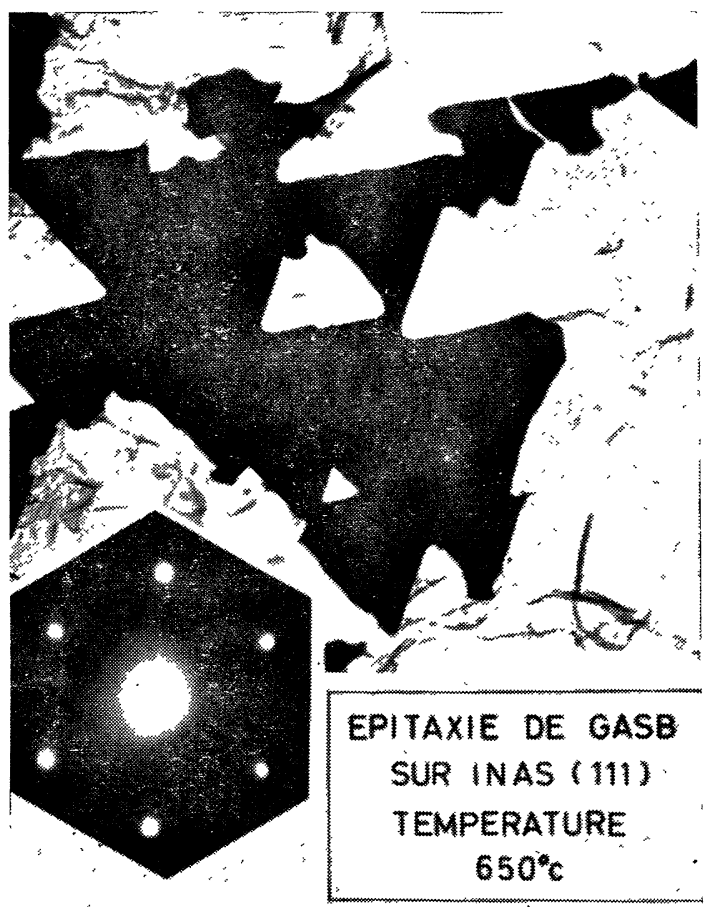

FIG. 10. - Microphotographie et diagramme de diffraction électronique d'un dépôt de GaSb sur InAs (111).

médaillon, est présentée une partie d'un diagramme de diffraction électronique de la même couche, démontrant l'identité de son orientation avec celle du substrat.

La méthode E. D. R. I. apparaît ainsi d'application assez générale ; elle présente cependant un certain nombre de limitations, dont la principale réside dans le fait que, de deux composés, on peut seulement déposer le plus volatil sur le moins volatil. Ainsi semble exclue l'isoépitaxie (dépôt d'un matériau sur un substrat du même matériau, dopé différemment, par exemple).

Il apparaît d'autre part que la méthode n'est applicable qu'aux couples de matériaux susceptibles d'interdiffuser. A titre d'exemple, il n'a pas été possible de faire croître de l'antimoniure d'indium sur du tellurure de cadmium car il n'existe pratiquement pas pour ce couple de solubilité mutuelle.

6. Conclusion. - Sous la réserve des limitations signalées, la méthode E. D. R. I. semble bien adaptée à la réalisation d'hétérojonctions utilisables dans des dispositifs électroniques à couches minces, tels que : photodétecteurs à large bande, photopiles, diodes lasers, diodes de commutations rapides.

Elle fournit d'autre part le moyen d'étudier la croissance de monocristaux dans le large domaine qui s'étend entre la couche dite mince (quelques dizaines d'angströms) et le monocristal massif, même pour des matériaux dont la volatilité a été jusqu'à présent un handicap lourd à surmonter. Le caractère isotherme de la méthode devrait permettre de simplifier l'interprétation théorique des phénomènes fondamentaux mis en jeu dans l'épitaxie.

Remerciements. - Les auteurs expriment leur gratitude à $H$. Rodot et $\mathrm{R}$. Triboulet pour la fourniture des matériaux, au C. N. E. T. pour avoir mis à leur disposition une microsonde électronique, à Mlle Dufour pour les mesures effectuées sur cette microsonde, ainsi qu'à C. Sella, du Laboratoire des Rayons X du C. N. R. S. pour l'étude des structures en microscopie et diffraction électroniques.

Manuscrit reçu le 28 juillet 1965.

\section{BIBLIOGRAPHIE}

[1] Bailly (F.), Marfaing (Y.) et Cohen-Solal (G.), J. Physique,

[2] Robinson (P. H.), R. C. A. Rev., 1963, 24, 574.

[3] Tannenbaum Handelman (E.) et Povilonis (E. I.), J. Electrochem. Soc., 1964, 111, 201.

[4] Rodot (H.) et Henoc (J.), C. R. Acad. Sc., Paris, 1963, 256, 1954.

[5] Bailly (F.), Cohen-Solal (G.) et Marfaing (Y.), C. R. Acad. Sc., Paris, 1963, 257, 103.
[6] Marfaing (Y.), Cohen-Solal (G.) et Bailly (F.), Conf. Int. Phys. Semicond., Paris, 1964, 1245 et BaILly (F.), 1964, 258, 556.

[7] Woolley (J. C.) et Ray (B.), J. Phys. Chem. Sol., 1960, 13, 151.

[8] Cohen-Solal (G.) et Marfaing (Y.), C.R. Acad.Sc., Paris, 1965, 260, 4191.

[9] Warekois (F. P.), Lavine (M. C.), Mariano (A. N.) et Gatos (H. C.), J. Appl. Phys., 1962, 33, 260. 